\title{
Propagating Neural Source Revealed by Doppler Shift of Population Spiking Frequency
}

\author{
Mingming Zhang, Rajat S. Shivacharan, Chia-Chu Chiang, Luis E. Gonzalez-Reyes, and Dominique M. Durand \\ Neural Engineering Center, Department of Biomedical Engineering, Case Western Reserve University, Cleveland, Ohio 44106
}

Electrical activity in the brain during normal and abnormal function is associated with propagating waves of various speeds and directions. It is unclear how both fast and slow traveling waves with sometime opposite directions can coexist in the same neural tissue. By recording population spikes simultaneously throughout the unfolded rodent hippocampus with a penetrating microelectrode array, we have shown that fast and slow waves are causally related, so a slowly moving neural source generates fast-propagating waves at $\sim 0.12 \mathrm{~m} / \mathrm{s}$. The source of the fast population spikes is limited in space and moving at $\sim 0.016 \mathrm{~m} / \mathrm{s}$ based on both direct and Doppler measurements among 36 different spiking trains among eight different hippocampi. The fact that the source is itself moving can account for the surprising direction reversal of the wave. Therefore, these results indicate that a small neural focus can move and that this phenomenon could explain the apparent wave reflection at tissue edges or multiple foci observed at different locations in neural tissue.

Key words: hippocampus; moving source; neural signal propagation

\section{Significance Statement}

The use of novel techniques with an unfolded hippocampus and penetrating microelectrode array to record and analyze neural activity has revealed the existence of a source of neural signals that propagates throughout the hippocampus. The source itself is electrically silent, but its location can be inferred by building isochrone maps of population spikes that the source generates. The movement of the source can also be tracked by observing the Doppler frequency shift of these spikes. These results have general implications for how neural signals are generated and propagated in the hippocampus; moreover, they have important implications for the understanding of seizure generation and foci localization.

\section{Introduction}

Propagation of neural activity is important for normal brain functions such as memory formation and normal sleep cycles, whereas abnormal neural propagation can result in pathological cases such as sleep disorders and epilepsy (Jarrard, 1993; Schwartzkroin, 1994; Lubenov and Siapas, 2009). One essential feature of neural propagation is that the complicated pattern of activity consists generally of different traveling waves. It has been shown that fast and slowly traveling waves coexist in both human and rodent animal brain tissue and that they propagate at differ-

\footnotetext{
Received Sept. 21, 2015; revised Jan. 19, 2016; accepted Jan. 28, 2016.

Author contributions: M.Z., L.E.G.-R., and D.M.D. designed research; M.Z. performed research; M.Z. and D.M.D. contributed unpublished reagents/analytic tools; M.Z., R.S.S., C.-C.C., and L.E.G.-R. analyzed data; M.Z., L.E.G.-R., and D.M.D. wrote the paper.

This work was supported by the National Institutes of Health (Grant 2R01NS060757-05A1). We thank B. Strowbridge and R. Galan of the Department of Neuroscience at Case Western Reserve University for helpful comments in reviewing this manuscript.

The authors declare no competing financial interests.

Correspondence should be addressed to Dr. Dominique M. Durand, Neural Engineering Center, Department of Biomedical Engineering, Wickenden Building 112, Case Western Reserve University, 2071 Martin Luther King Jr. Drive, Cleveland,0H 44106.E-mail:dxd6@case.edu.

DOI:10.1523/JNEUROSCI.3525-15.2016

Copyright $\odot 2016$ the authors $\quad 0270-6474 / 16 / 363495-11 \$ 15.00 / 0$
}

ent speeds (Haas and Jefferys, 1984; Schevon et al., 2012; Weiss et al., 2013). These studies indicate that the slowly propagating waves can modulate the shape of faster-propagating population spikes (Haas and Jefferys, 1984; Weiss et al., 2013). Another essential feature of neural propagation is that the firing activity often originates from a discrete area called the "source" or "focus" of propagation. Several studies have indicated the presence of multiple neural foci in both human subjects and animal models in both brain cortex and the hippocampus (Bertram, 1997; Sugiyama et al., 2009). These studies suggest that multiple neural foci arise simultaneously from several separate areas in the brain (Bertram, 1997; Schevon et al., 2008). In many of these cases, propagation of electrical activity seem to initiate in different locations and to propagate in multiple directions (Derchansky et al., 2006; Osawa et al., 2013; Patel et al., 2013). Researchers have shown that this multidirectional propagation takes place mainly along two dominant directions $180^{\circ}$ apart in both brain cortex and the hippocampus (Derchansky et al., 2006; Rubino et al., 2006; Takahashi et al., 2011; Osawa et al., 2013). Mapping the neural propagation and localizing the neural foci are usually difficult, but the identification of these neural sources and the study of the propagation have mechanistic, diagnostic, and therapeutic 

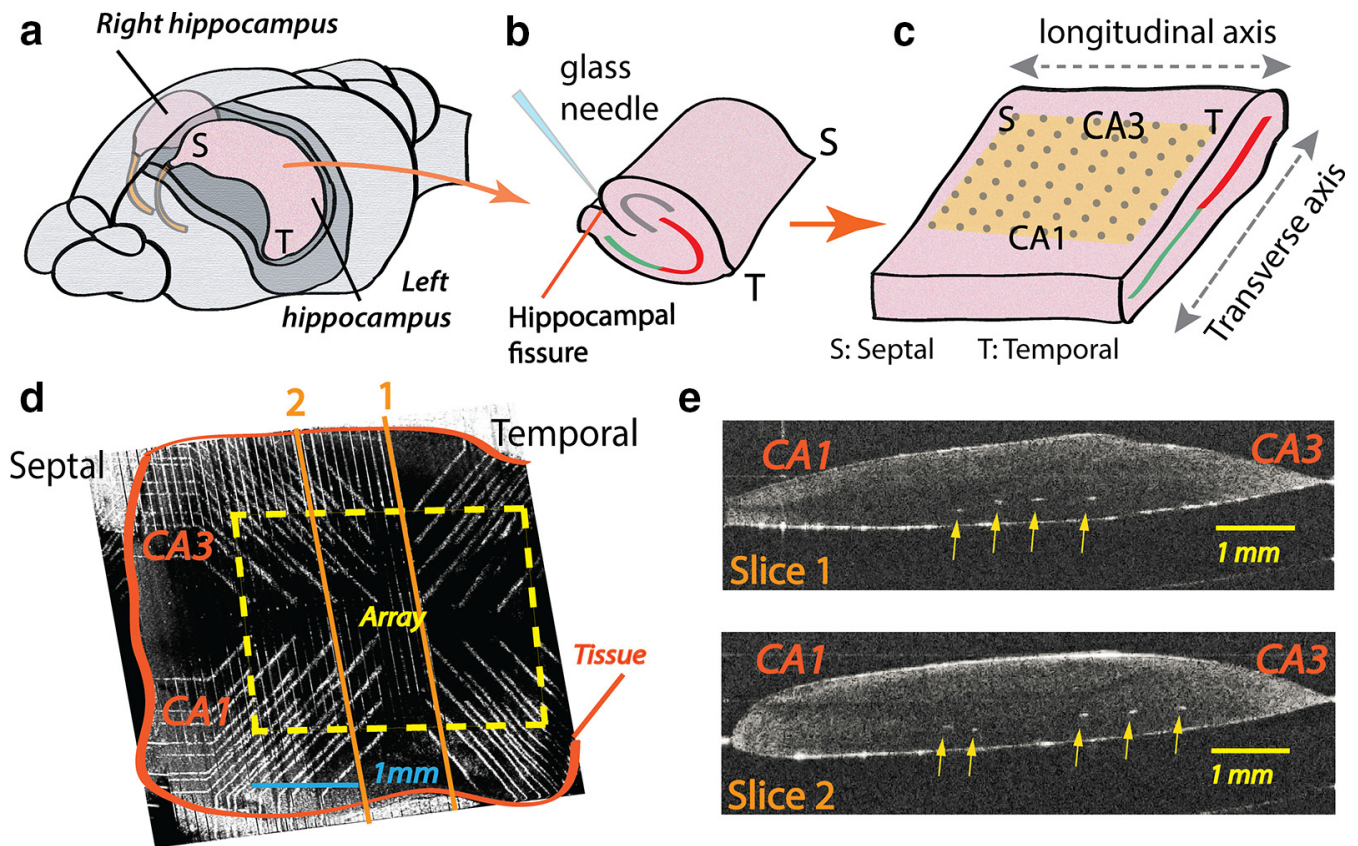

e
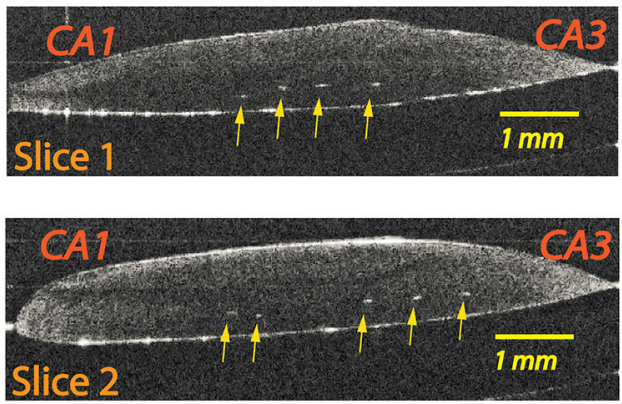

Figure 1. Surgery protocol for preparing the unfolded hippocampus preparation. $\boldsymbol{a}$, The left hippocampus was dissected from the septal-temporal lobe. $\boldsymbol{b}, \boldsymbol{c}$, A sharp glass needle was used to cut off the perforant path along the hippocampal fissure ( $\boldsymbol{b}$ ) and the hippocampus was unfolded by pulling over the Dentate Gyrus (DG) to further flatten the folded curved structure ( $\boldsymbol{c}$. The DG was cut off, leaving both CA3 and CA1 in the final preparation. $\boldsymbol{d}$, After unfolding, the hippocampus was positioned with the alveus at the bottom and placed onto the PMEA. OCT image shows that the unfolded hippocampus covered the PMEA. $\boldsymbol{e}$, Transverse scanning of the tissue in different positions along the longitudinal directions shown in $\boldsymbol{d}$. The white spots in the figure indicated by the arrows are the locations of the microelectrode tips. The dark lines in the tissue show the pyramidal cell layers. These figures are adapted from our previous publications; please refer to them for more information (Zhang et al., 2014; Zhang et al., 2015).

implications. For example, in the epilepsy clinic, patients with epileptiform propagation and multisite neural foci in temporal lobe are a frequent challenge and the mechanism is unknown (Pittau et al., 2014).

To understand how neural activity originates from multiple sites and propagates across the hippocampus, we have developed a preparation with the unfolded hippocampus and penetrating microelectrode array (PMEA) that enable us to detect neural propagation in the intact laminar architecture of the hippocampus (Zhang and Durand, 2011; Kibler et al., 2012; Zhang et al., 2014). This system makes it possible to monitor spatiotemporal activity in the flat hippocampal neural network in two dimensions and analyze the origin, direction, and speed of propagation with 64 individual channels in a PMEA (see Figs. 1, 2). Using this new technique, the data presented here describe a novel mechanism of propagation consisting of two different phenomena that governs the neural propagation: (1) a small, moving source traveling at a low speed and (2) a source that generates population spikes along its path that in turn propagate at a higher speed previously characterized as nonsynaptic propagation (Zhang et al., 2014).

\section{Materials and Methods}

\section{Experimental procedures and tissue preparation}

The protocol details were reviewed and approved by the Institutional Animal Care and Use Committee at Case Western Reserve University. CD1 mice of either sex from Charles River at postnatal day 10 (P10)-P20 were used. The unfolded hippocampus was prepared by following the surgical procedure described previously (Zhang et al., 2014; Zhang et al., 2015). A single hippocampus was dissected from the temporal lobe of the brain and unfolded by custom-made fire-polished glass pipette tools and a metal wire loop (Fig. 1). The artificial CSF (aCSF) buffer consisted of the following (in mM): $\mathrm{NaCl} 124, \mathrm{KCl} 3.75, \mathrm{KH}_{2} \mathrm{PO}_{4} 1.25, \mathrm{MgSO}_{4} 2$, $\mathrm{NaHCO}_{3}$ 26, dextrose 10 , and $\mathrm{CaCl}_{2}$ 2. 4-Aminopyridine (4-AP) was added to the normal aCSF to reach a final concentration of $100 \mu \mathrm{M}$. The PMEA was custom designed and fabricated with 64 penetrating microelectrodes arranged in an $8 \times 8$ matrix format on a transparent glass substrate. A single microelectrode has a height of $200 \mu \mathrm{m}$ and a diameter of $20 \mu \mathrm{m}$ (Kibler et al., 2012). During the experiment, an unfolded hippocampus was placed on top of the array with microelectrodes penetrating and reaching at a level right below the pyramidal cell layer (Fig. 1e). Then, the unfolded hippocampus was incubated with 4-AP aCSF in the recording chamber for $\sim 5-6$ min to induce spontaneous activity. Each microelectrode was connected to a custom-made amplifier with a gain of 100 and band-pass filter from 1 to $4 \mathrm{kHz}$. Signals from the array were digitized by a DAP 5400a A/D system (Kibler et al., 2012).

\section{Propagation mapping and neural source localization}

The microelectrodes would often detect positive deflection because most of electrode tips are located right below the pyramidal cell layer (Fig. 2b3). Time series recordings for all of the channels were rearranged into an Eight by Eight by $N$ data matrix, where the Eight by Eight matrix corresponds to the spatial arrangement of the microelectrodes and $N$ refers to the length of each recording channel in time points. $N$ was set at values corresponding to a time window varying from 50 to $80 \mathrm{~ms}$ to truncate only one individual neural firing spike in each channel. Bad recording from a certain failure channel was interpolated based on the recording from its surrounding channels. For each individual recording channel, the original data were filtered with a low-pass filter at $100 \mathrm{~Hz}$ and the time point at which the maximum amplitude happens was extracted and stored in a 2D matrix called peak-time matrix (Fig. 2). The peak-time matrix with 64 pixels was then linearly interpolated by generating new pixels using the values surrounding the generated pixel, turning the peak-time matrix into a 256-pixel matrix. Then, isochrones were generated based on this 256-pixel 2D matrix to show the propagation wave front of a single neural firing event at different time points. The origin of a neural propagation was defined as a neural source and it was estimated based on the geometrical center of the earliest channels where neural firing maximum amplitude happens first in the peak-time matrix (Fig. 2). 

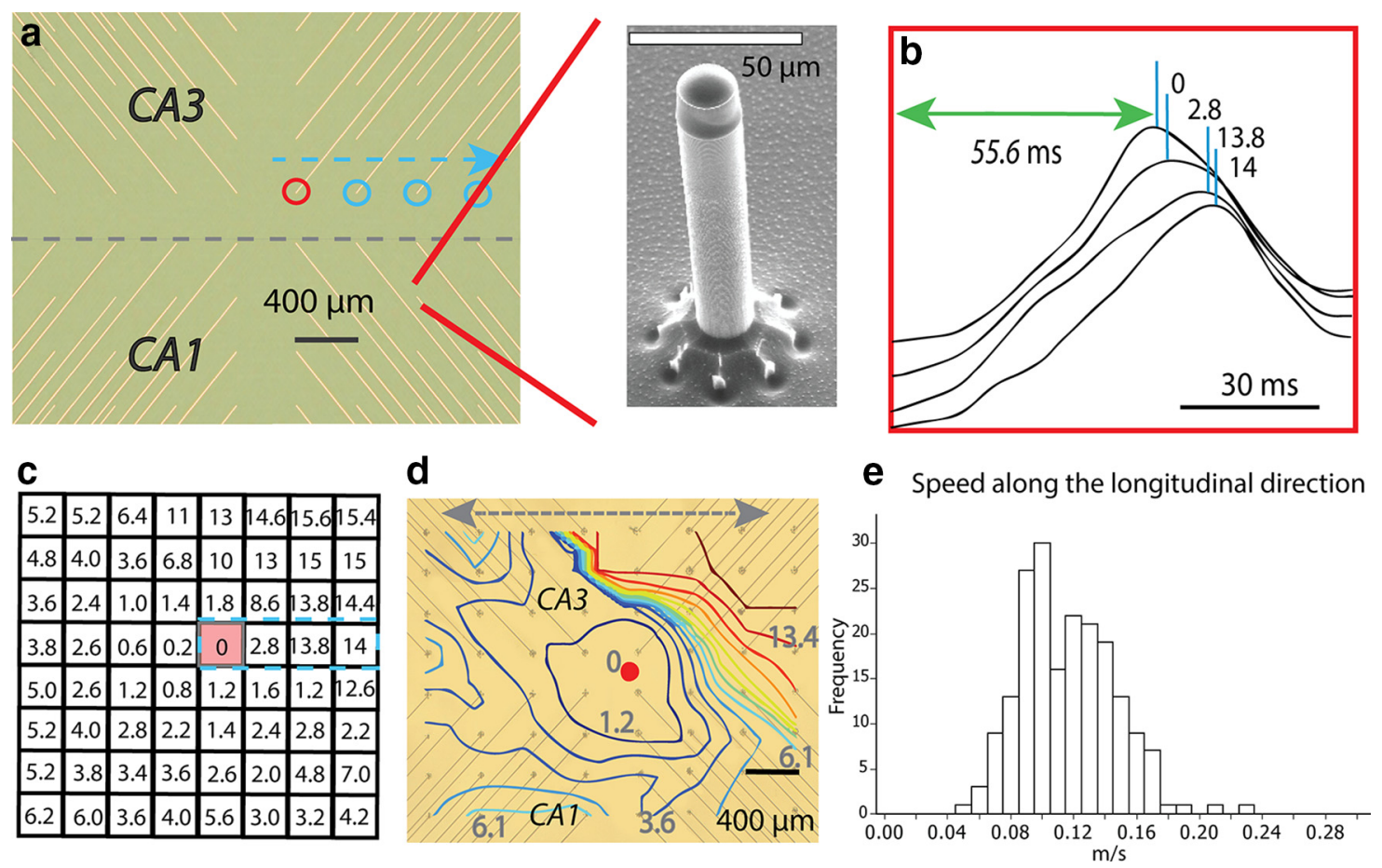

Figure 2. Recording obtained from a PMEA. $\boldsymbol{a}$, Image of the PMEA taken from under the array. The dashed line is in the region of CA3/CA1 border. The insertion on the right shows a single electrode with a diameter of $20 \mu \mathrm{m}$ and a height of $200 \mu \mathrm{m}$. $\boldsymbol{b}$, Neural spike propagation as recorded from four consecutive microelectrodes arranged linearly and marked in $\boldsymbol{a}$ (displayed in normalized amplitude and LPF at $100 \mathrm{~Hz}$ ). The delay between the first spike and the consecutive spikes are marked in milliseconds. c, Example of delays (in milliseconds) between the earliest peak and that from each of the 64 channels assigned to its corresponding pixel. The data are arranged in an $8 \times 8$ matrix and are defined as a peak-time matrix. $\boldsymbol{d}$, Isochrone map of the data presented in $\boldsymbol{c}$ showing a pattern of wave fronts corresponding to a single propagation event at different time points. The red dot indicates the earliest time when a firing peak occurs, which is defined as the source for a single neural propagation. The dashed line with arrowheads on the top indicates longitudinal direction. $\boldsymbol{e}$, Average propagation speed along the longitudinal axis was $0.12 \pm 0.03 \mathrm{~m} / \mathrm{s}$. The data shown here are from 194 speed calculations in seven different unfolded hippocampal preparations.

\section{Doppler effect estimation for a moving source}

Any moving source that generates propagating waves will produce a Doppler effect. To confirm whether the source for neural propagation was moving, Doppler equations were applied to the frequency of population spikes in front of and behind the source. For a source generating a propagating wave in a medium faster than the source itself, the observed frequency is given by $f=\left(\frac{c+v_{r}}{c+v_{s}}\right) f_{0}$ from Doppler effect, $f_{0}$ is the emitted frequency of the source, $v_{r}$ is the receiver speed, $v_{s}$ is the speed of the source moving, and $c$ is the velocity of the moving waves in the medium. Here, the velocity of the moving waves is the speed of the population spikes propagation, and its average speed is $c=0.12 \mathrm{~m} / \mathrm{s}$ (Zhang et al., 2014). The receiver is the array, so that $v_{r}=0$. Then, we have $f_{1}=\left(\frac{c}{c+v_{s}}\right) f_{0}, f_{2}=\left(\frac{c}{c-v_{s}}\right) f_{0}$, and $f_{1}, f_{2}$ are the observed instant frequency behind and in front of the moving source, respectively (see Fig. 8). Defining $R=\frac{f_{\text {front }}}{f_{\text {back }}}=\frac{f_{2}}{f_{1}}=\frac{c+v_{s}}{c-v_{s}}$ and speed of a moving source could be estimated based on the equation $v_{s}=\left(\frac{R-1}{R+1}\right) \times c$, where $R$ is the ratio of observed instantaneous frequency in front and behind the moving source. It is also possible to measure the distance moved by a single neural source and estimate the speed of a moving source based on the equation $v=d / t$ (see Fig. 8), where $d$ is the distance moved in a certain period of time $t$. Because the source is observed traveling across the array only during the first interspike interval in most cases (see Fig. 5), the Doppler estimation is applied only for these two population spikes. After the determination of the locations of source for the first and second propagation in a train, $d$ is estimated based the distance between these two locations (see Fig. 8). The time $t$ required for moving from the first to the second location was estimated by the time delay between the first spike peak in the train from the first channel and the peak of the second spike from the second channel (see Fig. 8b).

\section{Results}

To induce spontaneous activity in the unfolded hippocampus, an epileptogenic compound, 4-AP, was perfused in the recording chamber with aCSF at low doses of $100 \mu \mathrm{M}$ (Avoli and Perreault, 1987; Zhang et al., 2014). Spontaneous spike trains of activity induced by 4-AP were usually detected with variable numbers of individual population spikes in each train (number of spikes varying from one to five; Fig. 3).

Neural propagation shows a repeatable pattern: the direction of the first population spike is opposite to the propagation of the subsequent spikes in the same train

By analyzing the recording obtained from the 64 channels in the PMEA, the speed and propagation direction of each spike in the train could be measured (Zhang et al., 2014) (Fig. 2). The neural activity corresponding to each spike in the train propagates at an average speed of $0.12 \pm 0.03 \mathrm{~m} / \mathrm{s}$ (Fig. $2 e$ ) across the entire area of the unfolded hippocampus. Previous experiments have shown that direction of the propagation is preferentially longitudinal (septal to temporal or temporal to septal) (Zhang et al., 2014).

From the measurement of the time of arrival of population spikes at each recording site, isochrones maps are built and reveal the origin and direction of propagation in these spikes (red dots in Fig. 2d). Analysis of these maps indicates that the first spike in a train always originates in the temporal hippocampus. As a result, the propagation wave front moves longitudinally from the temporal to the septal region (74 of 82 trains, 17 different hippocampi). Surprisingly, the second spike in the train always originates in the septal region and propagates in opposite direction toward the temporal hippocampus (Fig. 3). The subsequent 

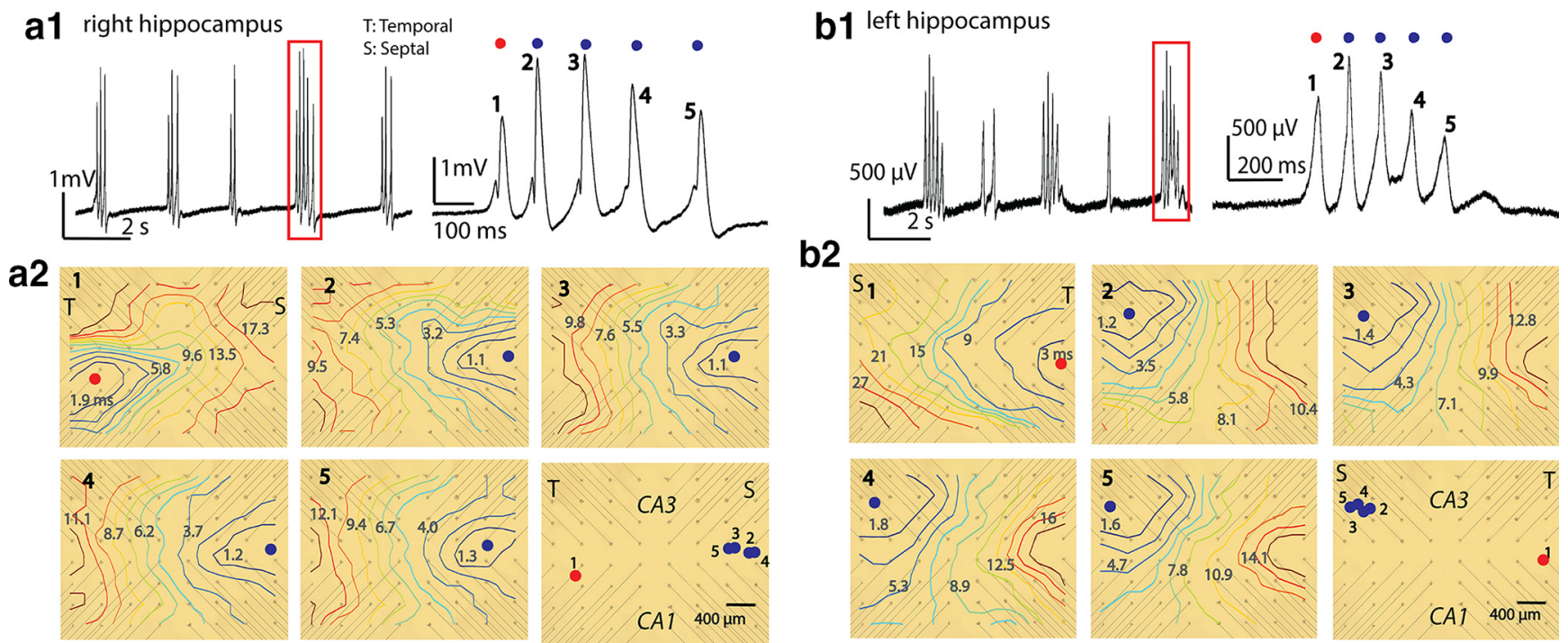

Figure 3. Source mapping for a single train propagating in the right and left hippocampi. The source of first spike in a train is located in the temporal pole, but the source of consecutive spikes in the same train is located in septal area. This result from the left hippocampus is consistent with that from the right hippocampus. $\boldsymbol{a} 1, \boldsymbol{b} \mathbf{1}$, Raw data from one microelectrode and the expanded view (red square) of a single spike train containing five spikes. $\boldsymbol{a} \mathbf{2}, \boldsymbol{b 2}$, Neural propagation patterns created by the peak-time matrix (also refer to the Materials and Methods). Each propagation event corresponds to a single spike from a microelectrode as shown in $\boldsymbol{a} \mathbf{1}$ and $\boldsymbol{b} 1$. Propagation of the first spike originates in the temporal area (source location indicated by a red dot), whereas the source of the consecutive spikes in the train is located in the septal area (blue dots).
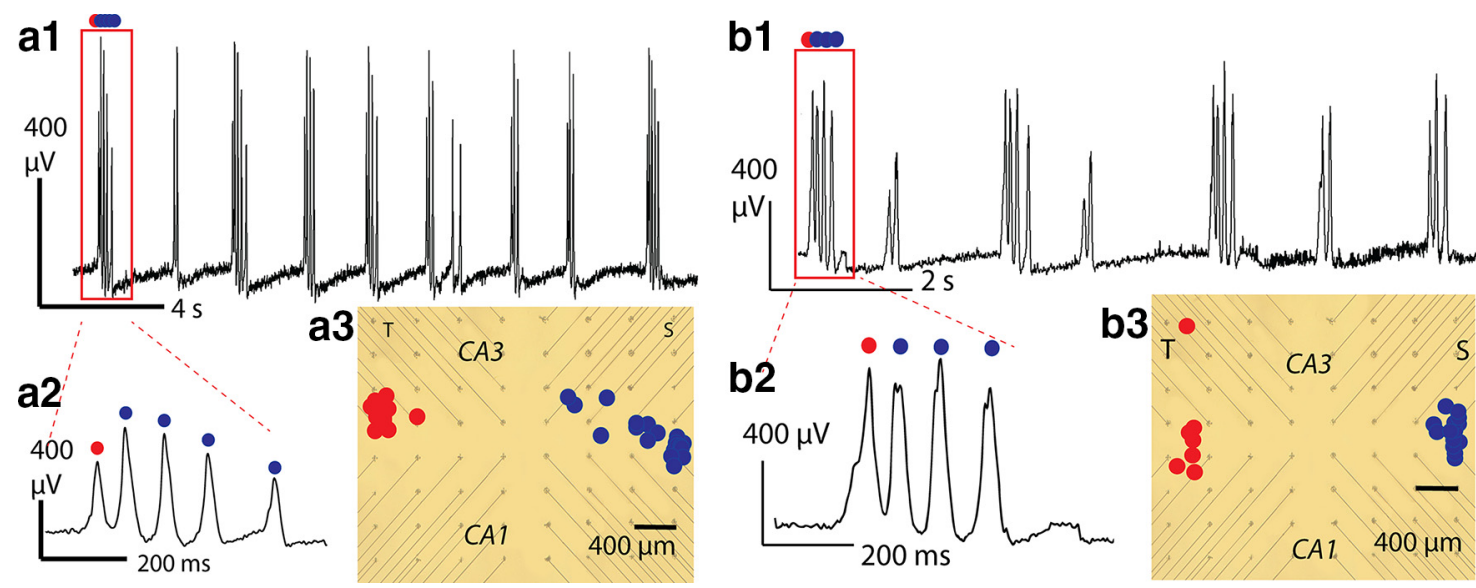

Figure 4. Source locations for multiple trains are consistent. The change of the source locations from the temporal to the septal pole is consistent across spike trains in the same and different hippocampi. $\boldsymbol{a}, \boldsymbol{b}$, Results from two different hippocampi. $\boldsymbol{a} \mathbf{1}, \boldsymbol{b} \mathbf{1}$, Raw data obtained from a single recording channel in the pMEA. $\boldsymbol{a} 2, \boldsymbol{b} 2$, Expanded view of the train showing the spike train from a time window marked as a red rectangle. $\boldsymbol{a} 3, \boldsymbol{b 3}$, Source locations for the first spike in all trains are located in the temporal area, whereas the source locations of the propagation for the consecutive spikes in each train are located in the septal area of the hippocampus.

spikes follow the same pattern as the second spike (Fig. 3a2,b2). Remarkably, this pattern of propagation is identical for all spike trains in the recording ( 82 of 82 ; see Fig. 5). For neural event with a single spike observed in some occasions ( 23 of 105 events), the location of the source is most likely located in the temporal lobe ( 9 of 11; see Fig. $5 c, d$ ). The direction of these single propagating spikes was the same as that described for the first spike in a train; that is, moving from the temporal to the septal hippocampus. These observations were confirmed when spikes were recorded from the same tissue ( $n=14$; Fig. 4 ) or from different hippocampi ( $n=81$ from 17 hippocampi; Fig. 5$)$ both ipsilaterally and contralaterally.

One possible explanation for this reversal of propagation is that the septal area is at the boundary of the hippocampus, which in turn generates another event propagating in the opposite direction (blue dot in Fig. 5). This explanation makes two predictions: (1) the secondary source should be located only at the boundary and (2) for sources (red dots) located in the middle of the tissue (Fig. 5), there should be no reversal of the propagation direction. To test these predictions, the size and the location of source of these population spikes must be first determined.

\section{Boundary effect cannot explain the propagation reversal}

The size and location of the source of the population spikes could be determined from the isochrones maps (Fig. 2d). Analysis of the data based on the information of the neural firing peaks indicate that the source of the propagating spike trains is confined to $1.52 \pm 0.98$ pixel size (an area of $\sim 0.18 \mathrm{~mm}^{2}$; Fig. 6). Given the small size of this source, it was possible to track its path in the tissue. The source of these spike trains first appeared preferentially in the temporal CA3 ( $n=36$, of 161 source locations, from 17 hippocampus; Fig. 5). For some spike trains, the origin of the neural propagation was found in the middle of the tissue ( $n=8$ of 81 trains), but the sources for all other spikes within the same 


\section{Detailed analysis for hippocampi from right and left side of the brain}
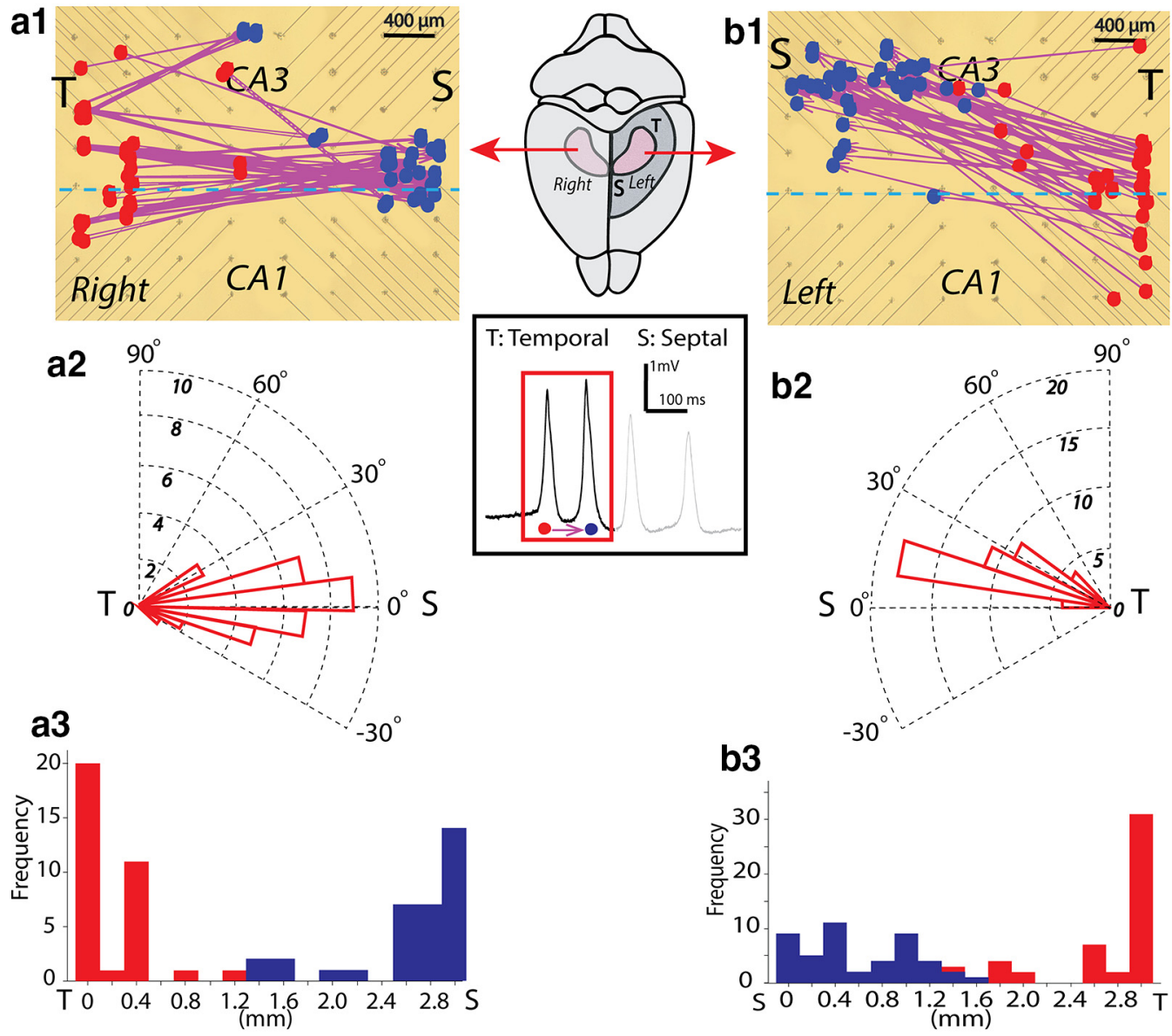

\section{Source locations for a single propagation in each group}

C

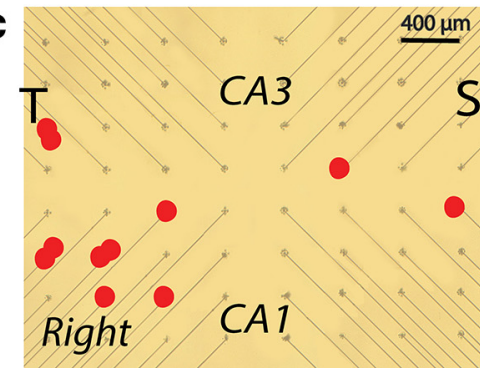

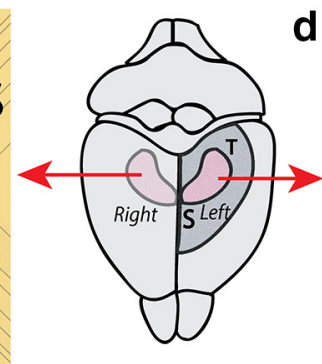

d $\mathrm{S}$

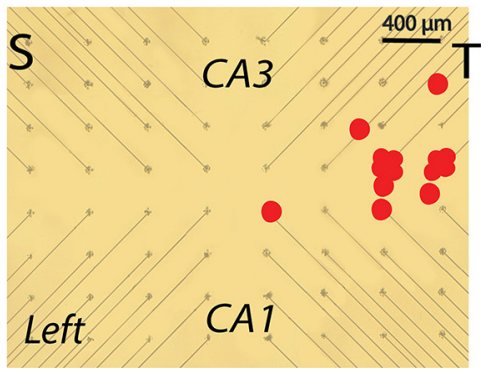

Figure 5. Spatial extent, locations, and moving directions of the sources. The pattern of propagation is consistent in every spike train recorded: the source of the first propagating spike is located in the temporal side and then shifts to the septal area for the consecutive spikes (Figs. 3, 4). Only the locations of the first and second spikes in each group are shown here. Information related to the source of the first propagation is marked in red and that of the second propagation in blue. The analysis was broken down into two groups: the right hippocampus and the left hippocampus. The spatial extent, location, and direction of the moving sources are anatomically consistent in hippocampi on both sides of the brain. The insertion below the rodent brain diagram shows a sample recording with a group of four neural spikes inside. Because in $98.7 \%$ of the neural spike trains ( 81 of 82 ) the location of the source for the second and subsequent spikes in a group remains in the septal area, only the locations of the source for the first two propagating spikes in a group are plotted in this figure. The first column (a1-a3) shows the data obtained from the right hippocampus and the second column (b1-b3) shows that from the left hippocampus. For the right hippocampus, the data shown here are from 35 different groups of neural firing train in seven different unfolded hippocampal preparations. For left hippocampus, the data are from 47 different trains of neural firing spikes in 10 different unfolded hippocampi. $\boldsymbol{a} \mathbf{1}, \boldsymbol{b} \mathbf{1}$, Locations of the neural propagation sources for all the first and second neural propagating spikes in different spikes trains. $\boldsymbol{a} \mathbf{2}, \boldsymbol{b} \mathbf{2}$, Direction in which the location of the sources has moved from each first propagating spike to its second location from the same firing train. $\boldsymbol{a} \mathbf{3}, \boldsymbol{b} \mathbf{3}$, Distance from all the locations of the neural sources to the edge of the recording array. The histogram confirms that the neural sources for all the first propagation reside mainly in the temporal hippocampus, whereas those for the second spikes occur mainly in the septal area of the hippocampus. There are several cases (the red histogram bar in the middle, $n=8$, of 82 trains) showing that the first neural spike in a train could originate in the middle of the tissue instead of temporal side. $\boldsymbol{c}, \boldsymbol{d}$, Location of the source for a single neural train, which means there is only a single neural spike in each train. The neural propagation could start anywhere in the tissue, but with a preference to originate in the temporal hippocampus. Nine of $11 \mathrm{neural}$ propagating spikes (from five different tissues) shown in coriginate from temporal area of the hippocampus, which is from the right side of the brain. All 14 spikes (from eight different tissues) start in the temporal hippocampus, which is from the left side of the brain hemisphere shown in $\boldsymbol{d}$. 
a Estimated size of the neural propagation sources
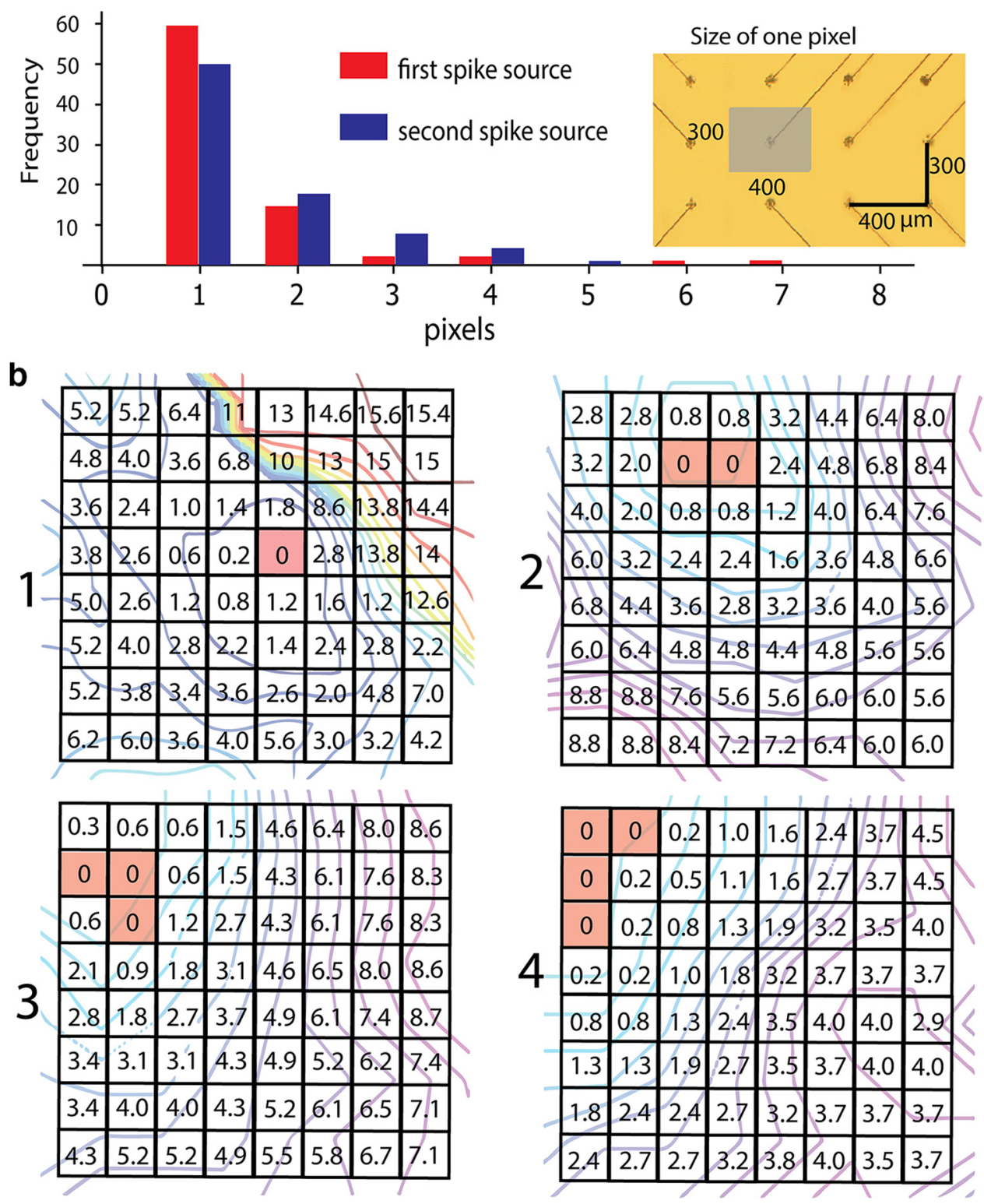

Figure 6. Method for size estimation of a source. $a$, The insert on the right side shows definition of one pixel area. The microelectrodes along the $x$-axis are $400 \mu \mathrm{m}$ apart from each other, whereas the distance between two of them along the $y$-axis is $300 \mu \mathrm{m}$. One pixel size is defined as a rectangular area of $300 \times 400 \mu \mathrm{m}$ based on the array's spacing information (the gray area), which is $0.12 \mathrm{~mm}^{2}$. The histogram shows that most of the sources have a size in a range of 1 or 2 pixels. The size of a source could be variable from 1 to 7 pixels in all experiments. The average size of the sources is $1.52 \pm 0.98$ pixels $\left(0.18 \pm 0.12 \mathrm{~mm}^{2}\right) \cdot \boldsymbol{b}$, Four different examples of the neural sources marked in different locations with different sizes. Isochrones in the background are the wave fronts of the propagation at different time points. In each propagation, the channel with the earliest peak time is marked in red here to mark the location of the source. In each example, the geometric center of the earliest channel or multiple channels is defined as the location of a neural propagation source.

train were always located in the septal side of the hippocampus (Fig. 5a1,b1). The source was then located in the septal area in the longitudinal direction and stayed in the septal area until the end of the train $(n=81$ of 81$)$. In some experiments, the source was observed in three different locations along its path (Fig. 7), indicating that the source was moving unidirectionally from the temporal to the septal hippocampus. These data clearly show that neither prediction made by the boundary effect is true and that the septal boundary itself is not required to produce the direction reversal. We then tested the hypothesis that the source itself is moving across the tissue, thereby generating spikes moving in the reverse direction.

\section{Reversal of propagation direction can be explained by a} moving source

By measuring the time delay and distance between the appearances of the source in the temporal and the septal regions, the source was observed to move and the propagation speed of the source was measured to be $0.016 \pm 0.007 \mathrm{~m} / \mathrm{s}$ (Fig. $8 d$ ). The speed of the source is significantly lower than that of the individual population spikes $(p<0.001)$. The source could be either jumping from one location to another or moving continuously across the tissue. If the source is continuously moving while generating the train of pulses, a Doppler effect should be observed in which the instantaneous frequency in front of the source $f_{2}$ 
Prop. 1 Prop. 2 Prop. 3

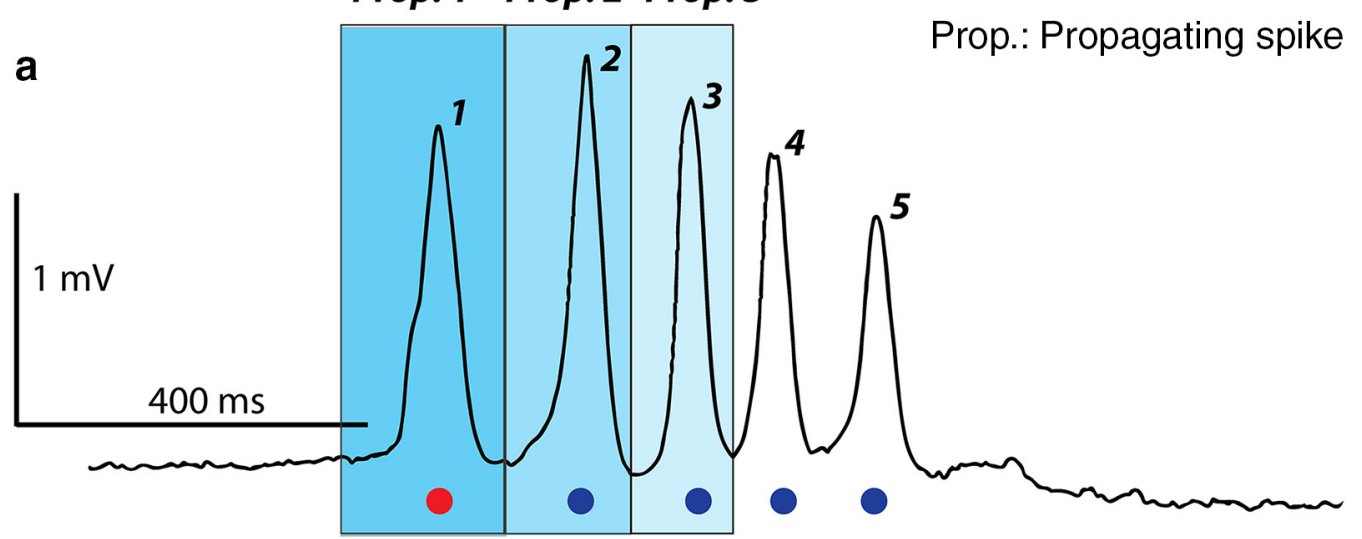

b
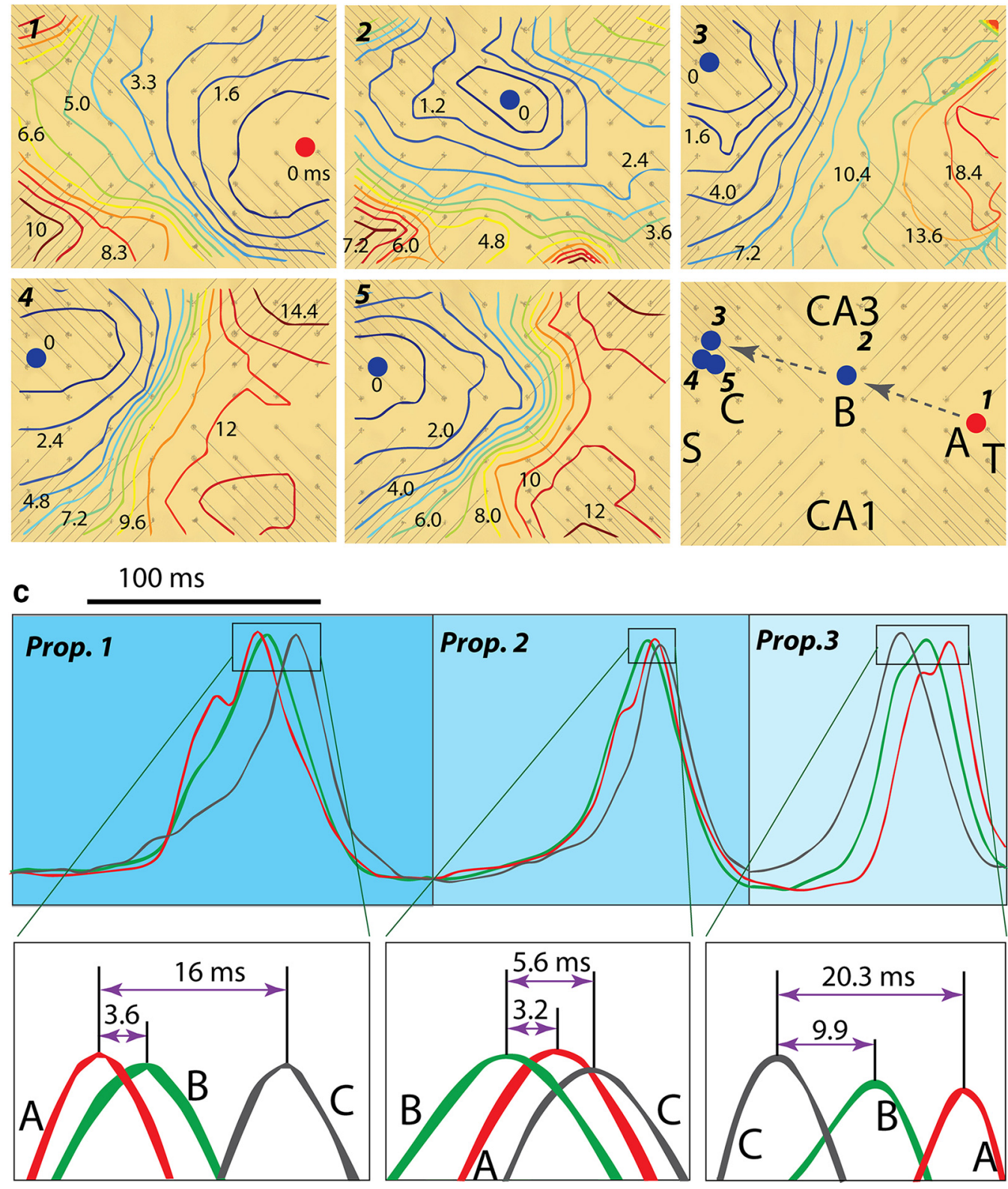

Figure 7. Moving source across the hippocampus. $\boldsymbol{a}$, High signal-to-noise ratio recording obtained from a single recording site showing a train with five spikes. The neural source locations from each spike (1-5) are marked with corresponding colored dots. $\boldsymbol{b}$, Neural foci location as expected from the isochrone maps. Various locations are mapped in the bottom picture. The first focus detected at location $A$ in the temporal side, then at location B in the middle of the tissue, and finally settles around location C in septal area of the hippocampus. The source speed in this example $(0.0092 \mathrm{~m} / \mathrm{s})$ is somewhat lower than the average, allowing observation from location A to location B. The origin of neural propagation is observed to move along its path across the entire T-S axis. c, Neural recordings from these three different locations show that the neural activity is propagating. Here, all of the recordings are displayed in their normalized (Figure legend continues.) 
a
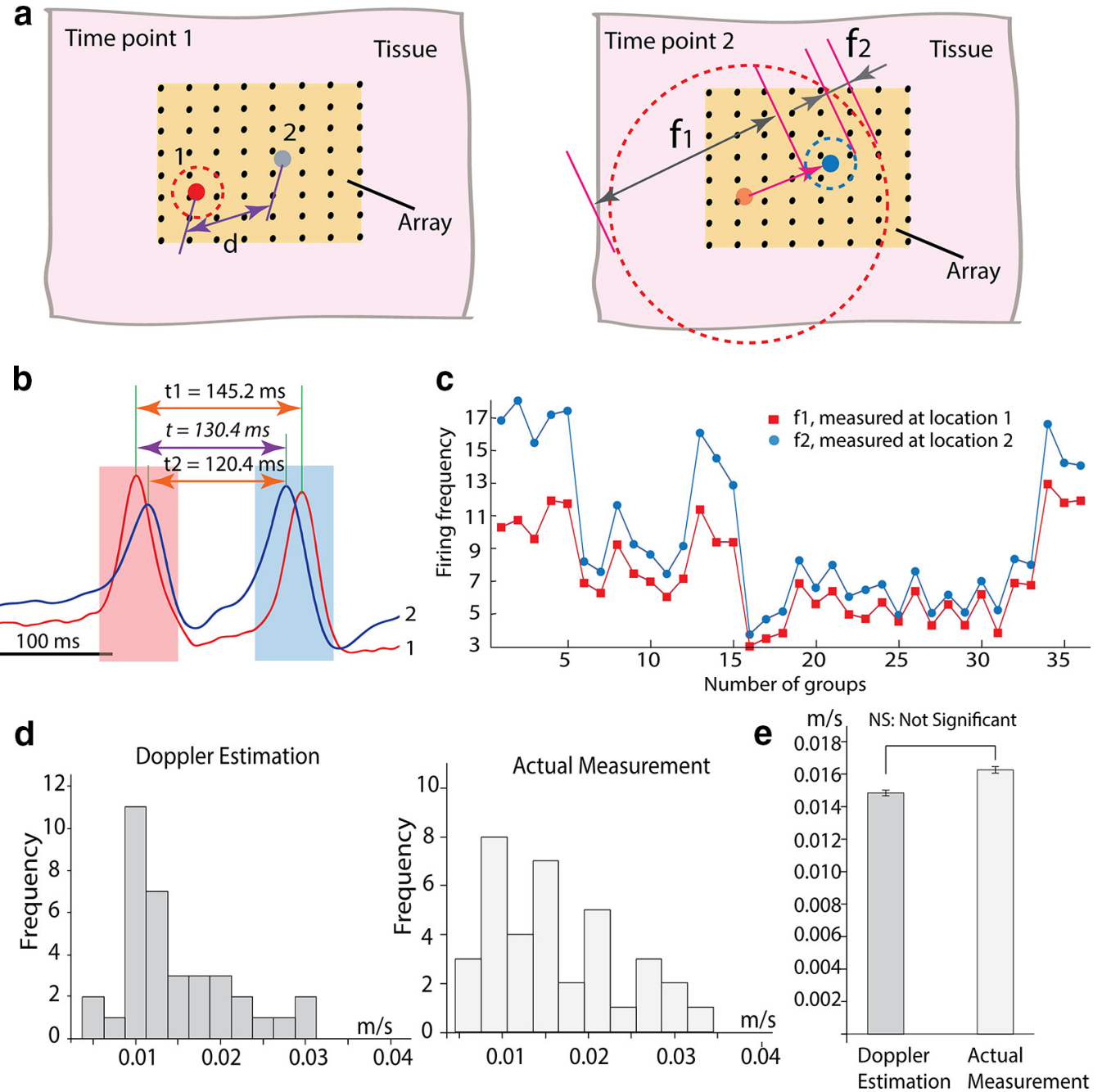

Figure 8. Actual measurement and Doppler-derived estimation of the speed of the source. $\boldsymbol{a}$, Schematic drawing showing how a moving source can generate propagation wave fronts at different time points with a distance traveled $(d)$, generating different observed instantaneous frequencies in front of $\left(f_{2}\right)$ and behind $\left(f_{1}\right)$ the moving source. $\boldsymbol{b}$, The two recordings shown here are from two channels situated near the first and second location of the source. These signals are displayed with normalized amplitude. The observed instant frequencies in front and behind the moving source is estimated from the measured time differences $t_{2}$ and $t_{1}$, respectively, from the spikes measured at location 1 and location 2 . $c$, Measurements of the instantaneous frequencies are based on 36 groups of neural activity (multiple neural firing spikes in each train) from eight different tissues. The results show that the frequency in front of the moving direction of the source $(9.71 \pm 4.46 \mathrm{~Hz}$ ) is significantly higher (paired $t$ test, $p<0.001$ ) than that behind the moving direction of the source $(7.37 \pm 2.84 \mathrm{~Hz}$ ). $\boldsymbol{d}$, Speed of the moving source is calculated using the Doppler equations and the actual measurement (distance/time). Please refer to Materials and Methods for details on the Doppler calculations. $\boldsymbol{e}$, Unpaired $t$ test shows that the speed estimated by the Doppler equations $(0.015 \pm 0.006 \mathrm{~m} / \mathrm{s})$ and the actual measurement $(0.016 \pm 0.007 \mathrm{~m} / \mathrm{s})$ are not significantly different.

should be significantly higher than the frequency of the spikes behind the source $f_{1}$. The instantaneous frequencies in front of and behind were measured and compared. The results are shown in Fig. 8 and indicate that $f_{2}$ (in front of the source) is significantly higher than $f_{1}$ (behind the source; $p<0.001$ ). Using the Doppler equation, the speed of the source could be estimated and was found to be $0.015 \pm 0.006 \mathrm{~m} / \mathrm{s}$. This value is not significantly different from the speed estimated by direct measurement (Fig. $8 d, e)$. These results show that the source is moving through the tissue at a lower speed as it is generating spike trains propagating at a faster speed in both directions.

$\leftarrow$

(Figure legend continued.) amplitudes. Red channel is from location A, green channel is from location $B$, and gray channel is from location C. Neural spike 1 is moving from location $A$ to $C$. Neural spike 2 is originating from location $B$ while moving from the middle to both sides of the hippocampus. Neural spike 3 shows that neural activity is moving from septal to temporal hippocampus with its source shown in location C. Please refer to Movie 1 for more details.

\section{Discussion}

Data here show that 4-AP-induced population spikes are generated by a localized source within the hippocampus. The source itself is moving at a speed of $\sim 0.016 \mathrm{~m} / \mathrm{s}$ and generates spikes at a frequency of $\sim 10 \mathrm{~Hz}$ traveling at a higher speed of $\sim 0.12 \mathrm{~m} / \mathrm{s}$. This phenomenon of a continuously moving source generating spikes along its path is consistent with the Doppler effect, in which the frequency in front of the source is higher than the frequency behind the source. 4-AP induces spontaneous spiking by blocking the Kv1 type of voltage-activated $\mathrm{K}^{+}$channels (Perreault and Avoli, 1992; Luhmann et al., 2000; Derchansky et al., 2006). Our analysis indicates the 4-AP-induced activity originates preferably in CA3 within a limited space. The average size of the source was estimated based on the dimension of PMEA and timing of the spikes plotted in isochrone maps. Population data show that the moving neural foci has an average limited size of $0.18 \pm 0.12 \mathrm{~mm}^{2}$ (Fig. 6). This result is consistent with those small neural foci reported in both human and animal brains 
(Bragin et al., 1999; Bragin et al., 2000; Bragin et al., 2005; Schevon et al., 2008).

The 4-AP-induced activity propagates longitudinally and transversely (Fig. 3). However, bilateral propagation in the longitudinal directions (temporal to septal or septal to temporal) is dominant, which is consistent with previous studies (Khalilov et al., 1997; Luhmann et al., 2000; Derchansky et al., 2006; Patel et al., 2013; Zhang et al., 2014). Bilateral propagation has been observed in human and nonhuman primate cortex, where neural activity propagates in two dominant directions $180^{\circ}$ apart ( $\mathrm{Ru}-$ bino et al., 2006; Takahashi et al., 2011). Here, the temporal hippocampus acts as the pacemaker to initiate trains of population spikes (Fig. 3). This is also consistent with previous literature showing that the temporal hippocampus is more epileptogenic under electrical or chemical stimulation (Gilbert et al., 1985; Bragdon et al., 1986; Derchansky et al., 2006). Surprisingly, analysis of the data shows a highly consistent propagation reversal (Fig. 4). The highly structured propagation pattern indicates that the first spike in a train always propagated in the temporal to septal direction, whereas the subsequent spikes propagated oppositely. This is very different from the seemingly random direction in the multidirectional propagation (Traub et al., 1993; Luhmann et al., 2000; Derchansky et al., 2006; Rubino et al., 2006; Takahashi et al., 2011).

Because the source is generally found near the septal or the temporal border, a possible explanation for the propagation reversal is the boundary effect. This was suggested in the monkeys' cortex, where waves were observed to reverse direction and the sulci generated a boundary (Rubino et al., 2006). However, this explanation is not consistent with these data because sources have also been found in the middle of the tissue (Fig. 5). In addition, in some experiments in which the speed was slightly slower and propagation could be observed for a longer distance $(n=9$ of 81 trains), the detection of multiple locations clearly showed that a source is moving from the temporal to the septal area (Fig. 7, Movie 1). Therefore, a single source travels from the temporal area along the longitudinal axis, generating propagating activity at different ends of the hippocampus along its trajectory within and beyond the region covered by PMEA. The fact that the source is only moving from the temporal to the septal hippocampus could be explained by the existence of functional and anatomical gradient along the septotemporal axis (Strange et al., 2014). The temporal hippocampus is bigger (Martens et al., 1998; Marx et al., 2013) and the pyramidal cell layer spreads out in the temporal area (Marx et al., 2013), leaving the temporal hippocampus with a bigger extracellular space. With the increasing gradient of NMDA receptor expression from the septal to the temporal hippocampus (Martens et al., 1998), the temporal area with higher excitability is more likely to become the initiation of the source. All of these septotemporal differences could explain why propagation preferentially initiates in the temporal hippocampus.

The source is not observed continuously because it can only be seen when it generates two traveling population spikes. Given the average speed of the source $(0.016 \mathrm{~m} / \mathrm{s})$, it takes $\sim 100-150 \mathrm{~ms}$ to travel through the length of the tissue. Because the spikes' average time interval is $\sim 121 \mathrm{~ms}$, in most cases, the source has already reached the septal area. This observation provides further evidence for the propagation reversal mechanism because, at the end of the first time interval, the source has reached the other side of the tissue and generates a pulse traveling in the reversed direction (Fig. 3).

Because the moving source is not observed continuously, it could be fixed but appear to jump from one location to the next,

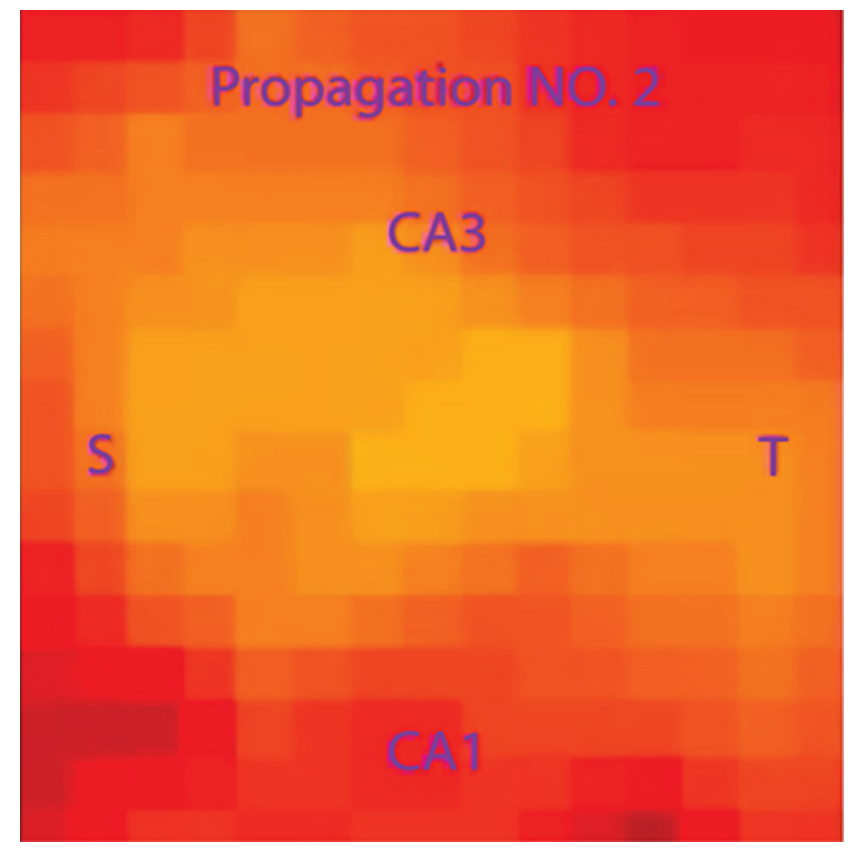

Movie 1. Neural propagation in a train of spikes from the hippocampus in right brain hemisphere, with the second source captured in the middle of the array. This movie presents the first three propagating spikes in a group of five and it is generated by applying individual normalization to each neural propagating spike (Zhang et al., 2014). In a single channel, the neural activity recording is characterized with five

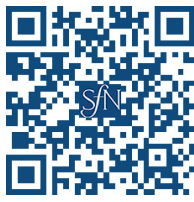
positive spikes in a group (Fig. 7). This video presents a different example with a similar pattern as shown in Figure 3. However, in this particular case, the second source located at the middle of the tissue clearly reveals the pathway of this moving source. Please refer to Figure 7 for more information.

similar to saltatory conduction in nerves. However, the moving sources can be described by the Doppler equation, suggesting a different explanation. A moving focus generating a sequence of firing pulses will generate a higher observed frequency in front of the source (Becker, 2011). Periodic oscillations and nonlinear finite waves generated by a continuously moving source can be described by the Doppler equations in the excitable conducting medium (Wellner et al., 1996; Brusch et al., 2003; Qian et al., 2010; Tranquillo et al., 2010). Here, the propagation of the source is described by this effect and the Doppler equation supports the hypothesis of a continuously moving source. The neural source generates a pulse approximately every $120 \mathrm{~ms}$ and the instantaneous frequency observed in front of the moving source is significantly higher than that observed behind the source. Because the speed calculated from the Doppler equations is similar to the direct measurement, the Doppler effect can be used to describe this phenomenon of a moving source. Therefore, the data can be accounted for by a Doppler effect, which rules out a fixed but jumping source because it assumes a continuously moving source (Sarkar et al., 2007). By measuring the distance that a source has moved and the time spent traveling, the average speed of the moving source was calculated $(0.016 \pm 0.007 \mathrm{~m} / \mathrm{s})$ and was almost 10 times slower than that of the neural activity propagation (0.12 $\pm 0.03 \mathrm{~m} / \mathrm{s}$; Fig. 8).

The slow movement of the source is consistent with potassium diffusion known to be nonsynaptic (Ghai et al., 2000). The speed of the moving source is $\sim 0.001-0.03 \mathrm{~m} / \mathrm{s}$ (Fig. $8 d$ ), which is consistent with the higher tail of the speed range from potassium diffusion (Lian et al., 2001; Durand et al., 2010). Potassium diffusion is ubiquitous in brain tissue and this mechanism could 
explain why there is a slowly moving source causing the change in direction of fast-propagating spikes in different parts of the brain (Bai et al., 2006; Rubino et al., 2006). Although consistent with diffusion, the source speed is somewhat higher than the average velocity of diffusion. This phenomenon could be caused by the hyperexcitability induced by the addition of 4-AP into the medium. Previous studies have shown that potassium-mediated slow propagation could reach a speed of $0.02 \mathrm{~m} / \mathrm{s}$ (Haas and Jefferys, 1984; Ghai et al., 2000). This propagation has been shown to take place through diffusive coupling in the interstitial space (Konnerth et al., 1986; Lian et al., 2001; Durand et al., 2010).

The coexistence of fast and slow waves during neural propagation has been described in both animal and human brains (Haas and Jefferys, 1984; Schevon et al., 2012). Data presented here provide an explanation for these different speeds of propagation (Haas and Jefferys, 1984; Schevon et al., 2012), showing that the slowly moving source is generating fast-propagating spikes along its path. Our results are consistent with data obtained in other preparations in which fast-propagating waves travel at a speed from 0.1 to $0.2 \mathrm{~m} / \mathrm{s}$, whereas the slow waves modulate the shape of fast spikes and propagate $\sim 10$ times more slowly (Haas and Jefferys, 1984; Rubino et al., 2006; Takahashi et al., 2011). However, the dynamics that govern the changes in the directions of fast propagation were unknown. By tracking the location of the source, we were able to show that the source is moving continuously. These data explain both the presence of fast and slow movement of activity and the propagation reversal in the brain (Rubino et al., 2006; Xu et al., 2007; Takahashi et al., 2011).

The observation of a slowly moving source could provide additional insights in the behavior of neural sources such as multiple epileptic foci often observed in the brain. These foci seem to be independent and uncorrelated and can be observed at different locations (Derchansky et al., 2006; Schevon et al., 2010). However, our analysis reveals a recurrent pattern of propagation along the septotemporal axis that could underlie the apparent randomness of neural foci locations. Data presented above suggest a different mechanism to explain some instances of multiple foci whereby a single unilateral focus could be generated by the same source, creating the appearance of multiple foci.

\section{Conclusion}

Spontaneous neural activity in the unfolded hippocampus can propagate with both slow and fast speeds simultaneously and with seemingly random direction of propagation. Here, we show that the propagation in the unfolded hippocampus follows a regular and consistent pattern. The first spike of the train propagates from the temporal to the septal pole at a speed of $0.1 \mathrm{~m} / \mathrm{s}$, whereas the other population spikes in the train were observed propagating in the opposite direction, from the septal to the temporal hippocampus. The analysis of spatial temporal patterns reveals that such a reversal in the propagation direction is a direct consequence of a single moving source (average speed of $0.016 \pm$ $0.007 \mathrm{~m} / \mathrm{s}$ ) detected by both direct and Doppler measurements. The observed speed of the moving source is consistent with the velocity previously reported for extracellular potassium waves. Therefore, a combination of slow and continuously moving sources generating fast-traveling spikes can explain the different speeds and change in the directions of neural propagation in the hippocampus.

\section{References}

Avoli M, Perreault P (1987) A GABAergic depolarizing potential in the hippocampus disclosed by the convulsant 4 -aminopyridine. Brain Res 400: 191-195. CrossRef Medline

Bai L, Huang X, Yang Q, Wu JY (2006) Spatiotemporal patterns of an evoked network oscillation in neocortical slices: coupled local oscillators. J Neurophysiol 96:2528-2538. CrossRef Medline

Becker BJ (2011) Unravelling starlight: William and Margaret Huggins and the rise of the new astronomy. Cambridge, MA: Cambridge University.

Bertram EH (1997) Functional anatomy of spontaneous seizures in a rat model of limbic epilepsy. Epilepsia 38:95-105. CrossRef Medline

Bragdon AC, Taylor DM, Wilson WA (1986) Potassium-induced epileptiform activity in area CA3 varies markedly along the septotemporal axis of the rat hippocampus. Brain Res 378:169-173. CrossRef Medline

Bragin A, Wilson CL, Engel J Jr (2000) Chronic epileptogenesis requires development of a network of pathologically interconnected neuron clusters: a hypothesis. Epilepsia. 41:S144-S152. CrossRef Medline

Bragin A, Engel J, Wilson CL, Fried I, Buzsáki G (1999) High-frequency oscillations in human brain. Hippocampus 9:137-142. Medline

Bragin A, Azizyan A, Almajano J, Wilson CL, Engel J Jr (2005) Analysis of chronic seizure onsets after intrahippocampal kainic acid injection in freely moving rats. Epilepsia 46:1592-1598. CrossRef Medline

Brusch L, Torcini A, Bär M (2003) Doppler effect of nonlinear waves and superspirals in oscillatory media. Phys Rev Lett 91:108302. CrossRef Medline

Derchansky M, Rokni D, Rick JT, Wennberg R, Bardakjian BL, Zhang L, Yarom Y, Carlen PL (2006) Bidirectional multisite seizure propagation in the intact isolated hippocampus: the multifocality of the seizure "focus." Neurobiol Dis 23:312-328. CrossRef Medline

Durand DM, Park EH, Jensen AL (2010) Potassium diffusive coupling in neural networks. Philos Trans R Soc Lond B Biol Sci 365:2347-2362. CrossRef Medline

Ghai RS, Bikson M, Durand DM (2000) Effects of applied electric fields on low-calcium epileptiform activity in the CA1 region of rat hippocampal slices. J Neurophysiol 84:274-280. Medline

Gilbert M, Racine RJ, Smith GK (1985) Epileptiform burst responses in ventral vs dorsal hippocampal slices. Brain Res 361:389-391. CrossRef Medline

Haas HL, Jefferys JG (1984) Low-calcium field burst discharges of CA1 pyramidal neurones in rat hippocampal slices. J Physiol 354:185-201. CrossRef Medline

Jarrard LE (1993) On the role of the hippocampus in learning and memory in the rat. Behav Neural Biol 60:9-26. CrossRef Medline

Khalilov I, Esclapez M, Medina I, Aggoun D, Lamsa K, Leinekugel X, Khazipov R, Ben-Ari Y (1997) A novel in vitro preparation: the intact hippocampal formation. Neuron 19:743-749. CrossRef Medline

Kibler AB, Jamieson BG, Durand DM (2012) A high aspect ratio microelectrode array for mapping neural activity in vitro. J Neurosci Methods 204:296-305. CrossRef Medline

Konnerth A, Heinemann U, Yaari Y (1986) Nonsynaptic epileptogenesis in the mammalian hippocampus in vitro. I. Development of seizurelike activity in low extracellular calcium. J Neurophysiol 56:409-423. Medline

Lian J, Bikson M, Shuai J, Durand DM (2001) Propagation of non-synaptic epileptiform activity across a lesion in rat hippocampal slices. J Physiol 537:191-199. CrossRef Medline

Lubenov EV, Siapas AG (2009) Hippocampal theta oscillations are travelling waves. Nature 459:534-539. CrossRef Medline

Luhmann HJ, Dzhala VI, Ben-Ari Y (2000) Generation and propagation of 4-AP-induced epileptiform activity in neonatal intact limbic structures in vitro. Eur J Neurosci 12:2757-2768. CrossRef Medline

Martens U, Capito B, Wree A (1998) Septotemporal distribution of [H3]MK-801, [H-3]AMPA and [H-3] Kainate binding sites in the rat hippocampus. Anat Embryol 198:195-204. CrossRef Medline

Marx M, Haas CA, Häussler U (2013) Differential vulnerability of interneurons in the epileptic hippocampus. Front Cell Neurosci 7:167. Medline

Osawa S, Iwasaki M, Hosaka R, Matsuzaka Y, Tomita H, Ishizuka T, Sugano E, Okumura E, Yawo H, Nakasato N, Tominaga T, Mushiake H (2013) Optogenetically induced seizure and the longitudinal hippocampal network dynamics. PLoS One 8:e60928. CrossRef Medline

Patel J, Schomburg EW, Berényi A, Fujisawa S, Buzsáki G (2013) Local generation and propagation of ripples along the septotemporal axis of the hippocampus. J Neurosci 33:17029-17041. CrossRef Medline 
Perreault P, Avoli M (1992) 4-aminopyridine-induced epileptiform activity and a GABA-mediated long-lasting depolarization in the rat hippocampus. J Neurosci 12:104-115. Medline

Pittau F, Grouiller F, Spinelli L, Seeck M, Michel CM, Vulliemoz S (2014) The role of functional neuroimaging in pre-surgical epilepsy evaluation. Front Neurol 5:31. Medline

Qian YD, HX, Liao XH, Hu G (2010) Doppler instability of antispiral waves in discrete oscillatory reaction-diffusion media. Chin Phys B 19:6.

Rubino D, Robbins KA, Hatsopoulos NG (2006) Propagating waves mediate information transfer in the motor cortex. Nat Neurosci 9:1549-1557. CrossRef Medline

Sarkar S, Ghosh S, Ghosh SK, Collier A (2007) Role of transcranial Doppler ultrasonography in stroke. Postgrad Med J 83:683-689. CrossRef Medline

Schevon CA, Ng SK, Cappell J, Goodman RR, McKhann G Jr, Waziri A, Branner A, Sosunov A, Schroeder CE, Emerson RG (2008) Microphysiology of epileptiform activity in human neocortex. J Clin Neurophysiol 25:321-330. CrossRef Medline

Schevon CA, Weiss SA, McKhann G Jr, Goodman RR, Yuste R, Emerson RG, Trevelyan AJ (2012) Evidence of an inhibitory restraint of seizure activity in humans. Nat Commun 3:1060. CrossRef Medline

Schevon CA, Goodman RR, McKhann G Jr, Emerson RG (2010) Propagation of epileptiform activity on a submillimeter scale. J Clin Neurophysiol 27:406-411. CrossRef Medline

Schwartzkroin PA (1994) Role of the hippocampus in epilepsy. Hippocampus 4:239-242. CrossRef Medline

Strange BA, Witter MP, Lein ES, Moser EI (2014) Functional organization of the hippocampal longitudinal axis. Nat Rev Neurosci 15:655-669. CrossRef Medline

Sugiyama I, Imai K, Yamaguchi Y, Ochi A, Akizuki Y, Go C, Akiyama T, Snead OC 3rd, Rutka TT, Drake JM, Widjaja E, Chuang SH, Cheyne D, Otsubo H (2009) Localization of epileptic foci in children with intractable epi- lepsy secondary to multiple cortical tubers by using synthetic aperture magnetometry kurtosis Clinical article. J Neurosurg Pediatr 4:515-522. CrossRef Medline

Takahashi K, Saleh M, Penn RD, Hatsopoulos NG (2011) Propagating waves in human motor cortex. Front Hum Neurosci 5:40. Medline

Tranquillo JV, Badie N, Henriquez CS, Bursac N (2010) Collision-based spiral acceleration in cardiac media: roles of wavefront curvature and excitable gap. Biophys J 98:1119-1128. CrossRef Medline

Traub RD, Jefferys JG, Miles R (1993) Analysis of the propagation of disinhibition-induced after-discharges along the guinea-pig hippocampal slice in-vitro. J Physiol 472:267-287. CrossRef Medline

Weiss SA, Banks GP, McKhann GM Jr, Goodman RR, Emerson RG, Trevelyan AJ, Schevon CA (2013) Ictal high frequency oscillations distinguish two types of seizure territories in humans. Brain 136:3796-3808. CrossRef Medline

Wellner M, Pertsov AM, Jalife J (1996) Spatial Doppler anomaly in an excitable medium. Phys Rev E Stat Phys Plasmas Fluids Relat Interdiscip Topics 54:1120-1125. Medline

Xu W, Huang X, Takagaki K, Wu JY (2007) Compression and reflection of visually evoked cortical waves. Neuron 55:119-129. CrossRef Medline

Zhang M, Durand DM (2011) Mapping 4-AP induced epilepsy propagation with a micro-electrode array in intact hippocampus in-vitro. 5th International IEEE/EMBS Conference on Neural Engineering (NER): 621-624.

Zhang M, Kibler AB, Gonzalez-Reyes LE, Durand DM (2015) Neural activity propagation in an unfolded hippocampal preparation with a penetrating micro-electrode array. J Vis Exp 97.

Zhang M, Ladas TP, Qiu C, Shivacharan RS, Gonzalez-Reyes LE, Durand DM (2014) Propagation of epileptiform activity can be independent of synaptic transmission, gap junctions, or diffusion and is consistent with electrical field transmission. J Neurosci 34:1409-1419. CrossRef Medline 